\title{
Axillary Lymph Nodal Metastases from Thyroid Carcinoma: Report of 2 Cases with Review of Literature
}

\author{
Shashank Shekhar Singh ${ }^{1}$, Swathy Krishnan ${ }^{1}$, Nitheeshraj K. Tigapuram ${ }^{1}$, Ashwani Sood ${ }^{1}$, Swayamjeet Satapathy ${ }^{1}$, \\ Manish Rohilla ${ }^{2}$, Sindhu Tanigassalam ${ }^{1}$, and Bhagwant Rai Mittal ${ }^{1}$ \\ ${ }^{I}$ Department of Nuclear Medicine, Post Graduate Institute of Medical Education and Research, Chandigarh, India; and ${ }^{2}$ Department \\ of Cytopathology and Gynecological Pathology, Post Graduate Institute of Medical Education and Research, Chandigarh, India
}

\begin{abstract}
Thyroid carcinoma has an excellent long-term outcome for locoregional disease if adequately treated, but the outcome declines sharply if distant metastatic disease is present. Axillary lymph nodal metastases are unusual in thyroid carcinoma and have a poorer outcome, as they are usually associated with aggressive histopathologies, extensive locoregional disease, and distant metastatic disease. We report 2 cases of thyroid carcinoma with axillary lymph nodal metastases and their management, and we review the literature on such cases.
\end{abstract}

Key Words: thyroid carcinoma; axillary lymph node; metastases; radioiodine

J Nucl Med Technol 2021; 49:89-91

DOI: $10.2967 /$ jnmt.120.249128

hyroid carcinomas are indolent and have an excellent long-term prognosis in early stages; however, the prognosis declines sharply if there are distant metastases. Apart from the neck lymph nodes, metastasis typically occurs in the lungs, bones, liver, and brain (1) but rarely involves the axillary lymph nodes (ALNs). Here, we report 2 cases of a histologic variant of differentiated thyroid carcinoma having ALN metastasis (ALNM), and we review the literature on such cases. An independent ethics committee approved this retrospective report, and the requirement to obtain informed consent was waived (INT/IEC/2020/SPL-921).

\section{CASE REPORTS}

\section{Case 1}

A 69-y-old woman with right-sided neck swelling for $5 \mathrm{y}$ was found to have papillary thyroid carcinoma (PTC) on cytopathology and underwent total thyroidectomy, central neck dissection, and right internal jugular vein ligation. Histopathologic examination revealed a multicentric colum-

\footnotetext{
Received May 6, 2020; revision accepted Aug. 13, 2020.

For correspondence or reprints contact: Ashwani Sood, Department of Nuclear Medicine, Post Graduate Institute of Medical Education and Research, Sector 12, Chandigarh 160012, India.

E-mail: sood99@yahoo.com

Published online Oct. 5, 2020.

COPYRIGHT (c) 2021 by the Society of Nuclear Medicine and Molecular Imaging.
}

nar cell variant of PTC, with metastasis involving 14 of 19 lymph nodes. Her stimulated serum thyroglobulin level $(1,409 \mathrm{ng} / \mathrm{mL})$ was elevated, and her level of antithyroglobulin antibody was normal $(30 \mathrm{IU} / \mathrm{mL})$. Diagnostic ${ }^{131} \mathrm{I}$ whole-body scanning and SPECT/CT, followed by ${ }^{18} \mathrm{~F}-\mathrm{FDG}$ PET/CT, showed no iodine avidity but intense ${ }^{18} \mathrm{~F}-\mathrm{FDG}$ avidity in the right and left cervical nodes, the right ALN, and a right lung nodule $(\sim 2.0 \times 2.0 \mathrm{~cm})$ (Fig. 1). Ultrasound-guided fine needle aspiration cytopathology (FNAC) of the right ALN found cytopathology suggestive of metastatic PTC (Fig. 2).

${ }^{131}$ I whole-body scanning and SPECT/CT after empiric ${ }^{131} \mathrm{I}$ therapy $(\sim 150 \mathrm{mCi})$ showed faint tracer uptake in the right supraclavicular region. Thalidomide and a suppressive dose of levothyroxine was started in view of the negative findings on iodine scanning and the elevated thyroglobulin level. Six months later, she showed a substantial decline in the levels of serum thyroglobulin $(13.7 \mathrm{ng} / \mathrm{mL})$ and antithyroglobulin antibody $(<15 \mathrm{IU} / \mathrm{mL})$. Presently, she is asymptomatic on follow-up.

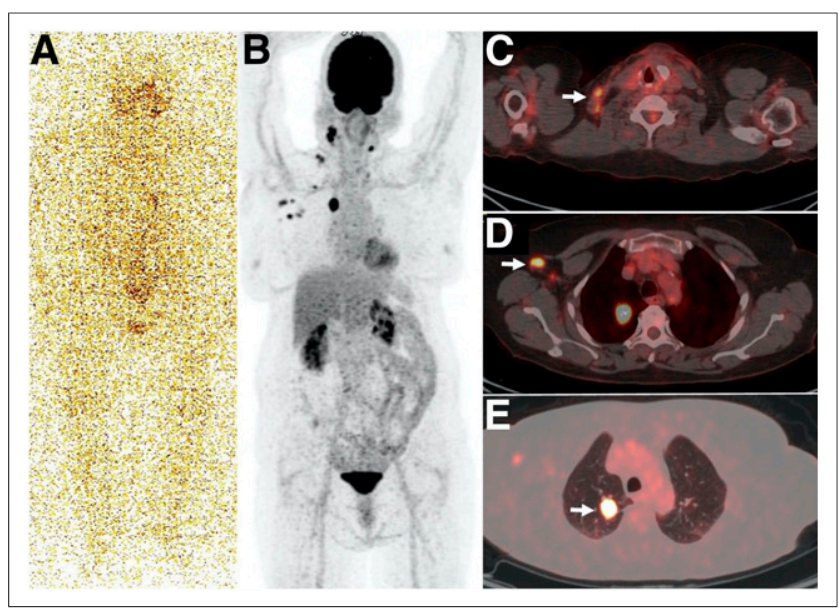

FIGURE 1. (A) Diagnostic whole-body ${ }^{131}$ I planar anterior image showing negative findings. (B) ${ }^{18} \mathrm{~F}-\mathrm{FDG} \mathrm{PET} / \mathrm{CT}$ maximumintensity-projection image showing abnormal foci of tracer uptake in bilateral cervical, right supraclavicular, right axillary and right thoracic regions. (C-E) Fused axial PET/CT images showing right cervical lymph nodes (C, arrow), axillary lymph nodes ( $D$, arrow), and right ALN, as well as right lung nodule (E, arrow). 


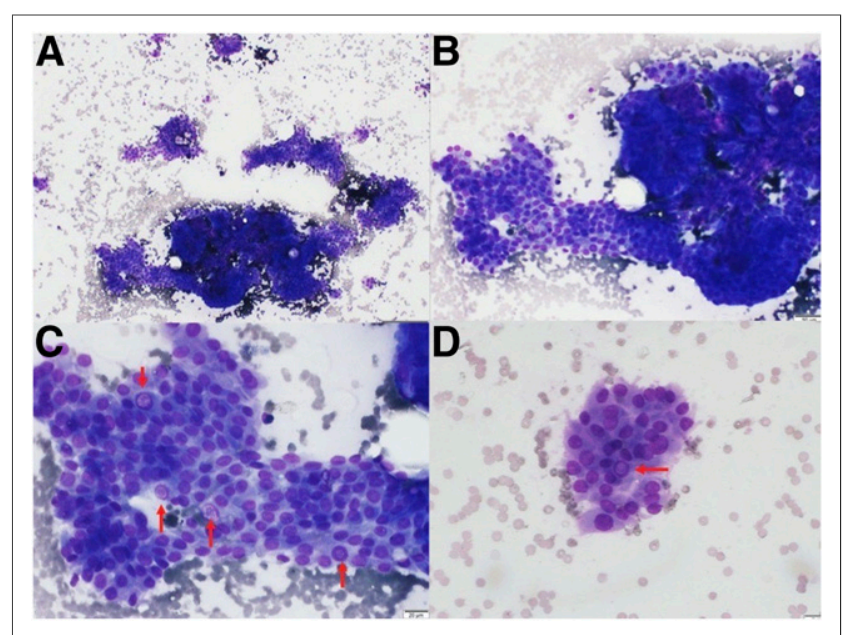

FIGURE 2. Fine-needle aspiration from right ALN yielded colloid mixed material and cellular smears. (A) Tumor cells arranged in papillae and small clusters (May GrunwaldGiemsa stain; $\times 40$ ). (B) Tumor papilla with rolled-up margins (May Grunwald-Giemsa stain; $\times 100$ ). (C and D) Individual tumor cells showing nuclear enlargement with focal crowding and prominent intranuclear pseudo inclusion (arrows; May Grunwald-Giemsa stain; ×200).

\section{Case 2}

A 70-y-old woman with midline neck swelling for 2 mo was found to have PTC on cytopathology and underwent total thyroidectomy and left selective neck dissection. Histopathologic examination revealed a tall cell variant of PTC with capsular invasion, a single intrathyroidal metastasis, and 3 metastatic cervical

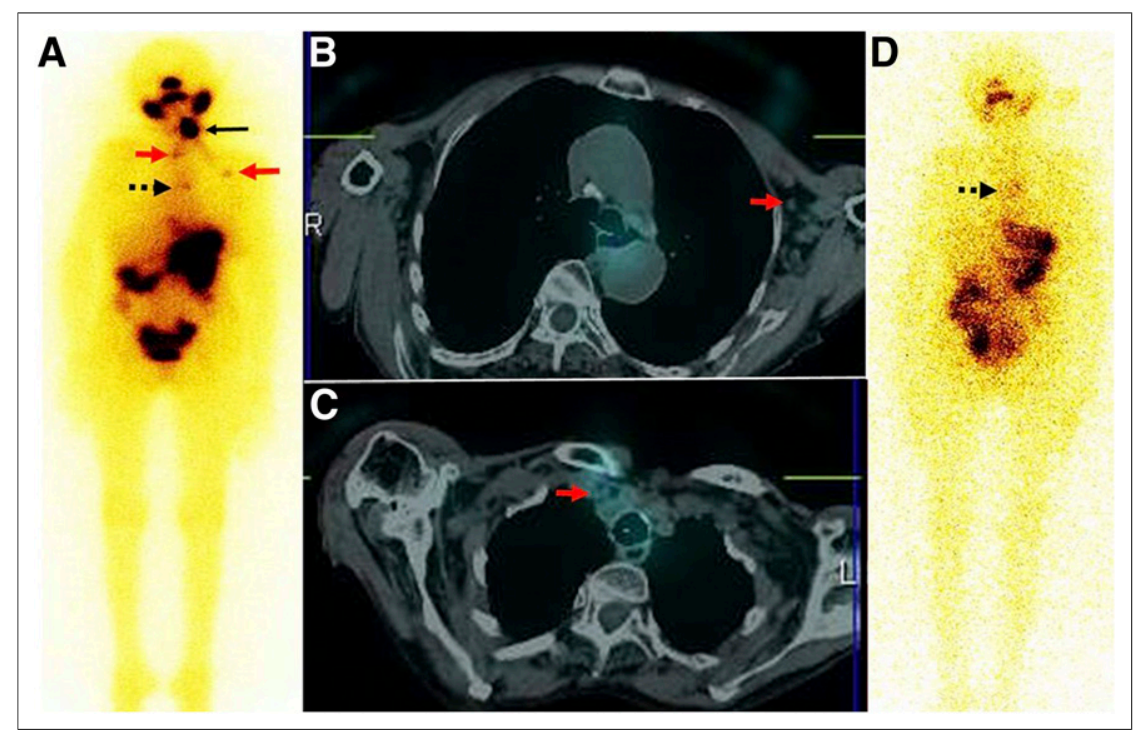

FIGURE 3. (A-C) Posttherapy whole-body ${ }^{131}$ I planar anterior image $(A)$ and fused axial SPECT/CT images (B and $C$ ) showing intensely tracer-avid remnant (solid black arrow), faintly tracer-avid left ALN, and highest mediastinal (red arrows) and prevascular (dashed arrow) lymph nodes. (D) Diagnostic whole-body ${ }^{131}{ }^{1}$ planar anterior image after 6 mo showing resolution of remnant and left ALN with faint tracer uptake in mediastinum (arrow). nodes $(4 / 20$ resected lymph nodes were positive for metastases).

In view of her raised stimulated thyroglobulin $(187 \mathrm{ng} / \mathrm{mL})$ and antithyroglobulin antibody $(19.5 \mathrm{IU} / \mathrm{mL})$, her intermediate risk-category, and the tracer-avid remnant on diagnostic ${ }^{131}$ I whole-body scanning, ${ }^{131}$ I therapy $(\sim 100 \mathrm{mCi})$ was administered. After therapy, iodine scanning and SPECT/ CT localized a tracer-avid remnant, a few of the highest mediastinal and prevascular nodes, and a single left ALN. After 6 mo, diagnostic ${ }^{131}$ I whole-body scanning showed no tracer uptake in the thyroid bed or the left axilla. There were a few foci of mediastinal tracer uptake, and the levels of stimulated serum thyroglobulin and antithyroglobulin antibody were reduced to $102 \mathrm{ng} / \mathrm{mL}$ and less than $15 \mathrm{IU} / \mathrm{mL}$, respectively (Fig. 3). A suppressive dose of levothyroxine was started because of the patient's advanced age and poor general condition. She succumbed to respiratory failure $19 \mathrm{mo}$ after the initial diagnosis.

\section{DISCUSSION}

A review of the literature found a total of 31 cases of histopathologically proven thyroid carcinoma with ALNM, including the 2 cases presented here (Supplemental Table 1; supplemental materials are available at http://jnmt.snmjournals. org).

Both sexes were almost equally affected (16 men, 15 women), and the mean age was 51.6 y (range, 19-69 y). The histology of the primary tumor was PTC in 21 patients, 8 of whom had a high-risk variant or a poorly differentiated component. The other histopathologic types included medullary thyroid carcinoma $(n=5)$, follicular thyroid carcinoma $(n=$

$2)$, mucoepidermoid carcinoma $(n=1)$, mucin-producing adenocarcinoma $(n=$ $1)$, and sclerosing mucoepidermoid carcinoma $(n=1)$ (only the last 3 mentioned are rare histopathologies).

Of the 31 patients, 24 and 1 presented with N1b and N1a disease, respectively. The nodal status was unknown in 5 patients, and 1 patient had no nodal involvement. Eleven patients had ALNM at the initial presentation (concurrent disease), and 19 had recurrent disease with a mean duration of 14.3 $y$ from the initial management (range, 3 mo to $41 \mathrm{y}$ ). The status of 1 patient in the study cohort was unknown.

Fourteen of the 31 patients had metastatic disease at the initial presentation, 4 of whom had the ALN as the only metastatic site, whereas 10 had additional metastatic lesions in the lung $(n=8)$, skeleton $(n=3)$, or liver $(n=2)$ or had pericardial effusion $(n=1)$. 
Most patients with a histologic variant or poorly differentiated thyroid carcinoma had ALNM at presentation with the primary disease or within a few months of presentation. However, most patients with PTC had ALNM as recurrent disease on follow-up. In 2 of the patients with initial PTC, aggressive recurrent PTC variants in the ALN were found on histopathology nearly $10 \mathrm{y}$ later, suggesting that well-differentiated PTC could have transformed into poorly differentiated carcinoma during the disease course $(2,3)$.

Postulated mechanisms for metastasis of thyroid carcinoma to the ALN may be direct communication between the cervical and axillary lymphatics, retrograde dissemination to regional lymphatic channels, or hematogenous dissemination $(4,5)$. Extensive cervical nodal metastases or previous neck dissection may favor altered lymphatic flow secondary to lymphatic blockage or fibrosis from prior surgery.

\section{CONCLUSION}

ALNM is detected on ${ }^{131} \mathrm{I}$ scanning or ${ }^{18} \mathrm{~F}-\mathrm{FDG}$ PET/CT, apart from conventional imaging. Radioiodine uptake in the ALN is usually synonymous with metastatic disease from differentiated thyroid carcinoma. Our review of the literature showed that most were managed with ALN dissection, although a few were treated with ${ }^{131}$ I for iodine-avid disease, with improved outcomes. Long-term follow-up is essential in these patients with unusual metastases involving the ALN.

\section{DISCLOSURE}

No potential conflict of interest relevant to this article was reported.

\section{REFERENCES}

1. Byar DP, Green SB, Dor P, et al. A prognostic index for thyroid carcinoma: a study of the EORTC Thyroid Cancer Cooperative Group. Eur J Cancer. 1979; 15:1033-1041.

2. Ozaki O, Ito K, Mimura T, et al. Anaplastic transformation of papillary thyroid carcinoma in recurrent disease in regional lymph nodes: a histologic and immunohistochemical study. J Surg Oncol. 1999;70:45-48.

3. Sakamoto A, Kasai N, Sugano H. Poorly differentiated carcinoma of the thyroid: a clinicopathological entity for a high-risk group of papillary and follicular carcinoma. Cancer. 1983;52:1849-1855.

4. Kowalski LP. Noncervical lymph node metastasis from head and neck cancer. ORL J Otorhinolaryngol Relat Spec. 2001;63:252-255.

5. Sherman SI. Thyroid carcinoma. Lancet. 2003;361:501-511. 